\title{
The Need for Novel Graphical Presentation of Cancer Data: Mushroom Cloud and Floating-Bar Plots
}

\author{
Jimmy T Efird ${ }^{1-3}$, Stephen W Davies ${ }^{4}$, Tithi Biswas ${ }^{5}$, Wesley T O'Neal ${ }^{6}$ and Ethan Anderson ${ }^{7}$ \\ ${ }^{1}$ Department of Public Health, Brody School of Medicine, East Carolina University, Greenville, NC, USA \\ ${ }^{2}$ Leo Jenkins Cancer Center, Brody School of Medicine, East Carolina University, Greenville, NC, USA \\ ${ }^{3}$ Center for Health Disparities, Brody School of Medicine, East Carolina University, Greenville, NC, USA \\ ${ }^{4}$ Department of General Surgery, University of Virginia School of Medicine, Charlottesville, VA, USA \\ ${ }^{5}$ Department of Radiation Oncology, University Hospitals, Case Medical Center, Case Western Reserve University School of Medicine, Cleveland, OH, USA \\ ${ }^{6}$ Department of Internal Medicine, Wake Forest University School of Medicine, Winston-Salem, NC, USA \\ ${ }^{7}$ Department of Pharmacology and Toxicology, Brody School of Medicine, East Carolina University, Greenville, NC, USA
}

\section{Introduction}

The introduction of the information age has created new opportunities for data collection, processing, and representation in the field of cancer research. Advances in bioinformatics, automated data retrieval systems, and data storage have improved data accessibility and the ability to make critical decisions based on the analyses of often complex and multivariable data. The challenge remains to condense and filter this data in such a fashion that it is readily interpretable to trained scientists, clinicians, the general public, and funding agencies (i.e., individuals with the most to gain from the information but with the least amount of time to interpret the data). These points are especially important if one's results are to be translated into practical application and the solicitation of future research support. The ultimate goal is to display information as simply as possible without losing the significant meaning and interpretation of the results.

\section{Current Graphical Methods and Techniques}

Typically scientific data is displayed as simple line plots or bar charts. While this is effective at condensing large amounts of data and satisfying the simplicity component, meaning may be lost, as these graphical methods often do not convey the underlying dynamics of the data.

\section{Next Generation Graphics}

Next generation graphics fill the above void by transforming single-dimensional displays, and sometimes lengthy and complex tables, into plots that convey multidimensional information in a precise but readily interpretable manner. In this paper, using data from a population-based study of lung cancer at a large tertiary referral hospital, we illustrate the advantage of presenting clinical results using next generation graphics.

\section{Data Source and Patient Population}

Patients who were evaluated and treated for lung cancer between 2001 and 2010 at the Leo Jenkins Cancer Center, East Carolina University were included in this analysis.

Data were obtained from the Vidant Medical Center Cancer Registry, which includes patients seen at Vidant Medical Center, East Carolina University Brody School of Medicine, Physicians East, SurgiCenter, and other local doctor's offices. The extracted information included data on age, race (self-reported), and insurance status. All histological types and stages of lung cancer were included. Only patients with private insurance were included in these analyses.

The eastern region of North Carolina (ENC) is a racially diverse population that has experienced historic differences in socioeconomic position and discrimination. The majority of the counties in ENC (97\%) fall below the national per capita income of $\$ 27,915$, with half reporting a value $<\$ 20,000$ [1]. Additionally, over $90 \%$ of the counties have a higher percentage of blacks than the national average of $13 \%$. In recent years, differences in socioeconomic status have shifted towards improved racial and economic equality, particularly among the younger and better educated generation.

\section{Hypothesis and Results}

We hypothesized that younger blacks with lung cancer are more likely to have private health insurance than their older generation counterparts.

Our data showed a decreasing linearly association between age and prevalence of private health insurance among black lung cancer patients (Table 1). In contrast, a reverse age trend was observed among white patients. These data suggest (but do not prove) that racial disparities in health insurance coverage among lung cancer patients in ENC may be slowly disappearing, possibly reflecting ongoing efforts aimed at improving the socioeconomic condition and education level among younger generation blacks in the region.

\begin{tabular}{|l|l|l|l|l|}
\hline Patient Age (Years) & White n(\%) & Black n (\%) & P-Value $\alpha$ & RR (95\%Cl) \\
\hline Q1 (25-62) & $203(18)$ & $220(38)$ & $<0.0001$ & 1.0 Referent \\
Q2 (63-68) & $286(26)$ & $137(24)$ & & $1.4(1.3-1.6)$ \\
Q3 (69-74) & $307(28)$ & $116(20)$ & & $1.5(1.3-1.7)$ \\
Q4 ( $\geq 75)$ & $320(29)$ & $102(18)$ & & $1.6(1.4-1.8)$ \\
& & & & $P_{\text {trend }}<0.0001^{*}$ \\
\hline Mean \pm SD & $70 \pm 9.5$ & $66 \pm 10$ & $<0.0001$ & -- \\
\hline Median (range) & $71(29-94)$ & $66(41-95)$ & & \\
\hline
\end{tabular}

* Likelihood ratio trend test

${ }^{\alpha}$ Tests of Statistical Significance (Fisher Exact for Categorical Variables, Exact Deuchler-Wilcoxon for Continuous Variables)

$\mathrm{Cl}=$ confidence interval, $\mathrm{Q} 1=$ first quartile, $\mathrm{Q} 2=$ second quartile, $\mathrm{Q} 3=$ third quartile $\mathrm{Q} 4=$ fourth quartile, $\mathrm{RR}=$ relative risk, $\mathrm{SD}=$ standard deviation.

Table 1: Characteristics and Relative Risk among Patients with Private Health Insurance.

*Corresponding author: Jimmy T Efird, Department of Public Health, Brody School of Medicine, East Carolina University, Physicians Quadrangle N, Greenville, NC 27834, USA, Tel: +1.650.248.8282; E-mail: jimmy.efird@stanfordalumni.org

Received August 22, 2013; Accepted August 24, 2013; Published August 26 , 2013

Citation: Efird JT, Davies SW, Biswas T, O'Neal WT, Anderson E (2013) The Need for Novel Graphical Presentation of Cancer Risk: Mushroom Cloud and Floating-Bar Plots. Trop Med Surg 1: e104. doi:10.4172/2329-9088.1000e104

Copyright: () 2013 Efird JT, et al. This is an open-access article distributed under the terms of the Creative Commons Attribution License, which permits unrestricted use, distribution, and reproduction in any medium, provided the original author and source are credited. 


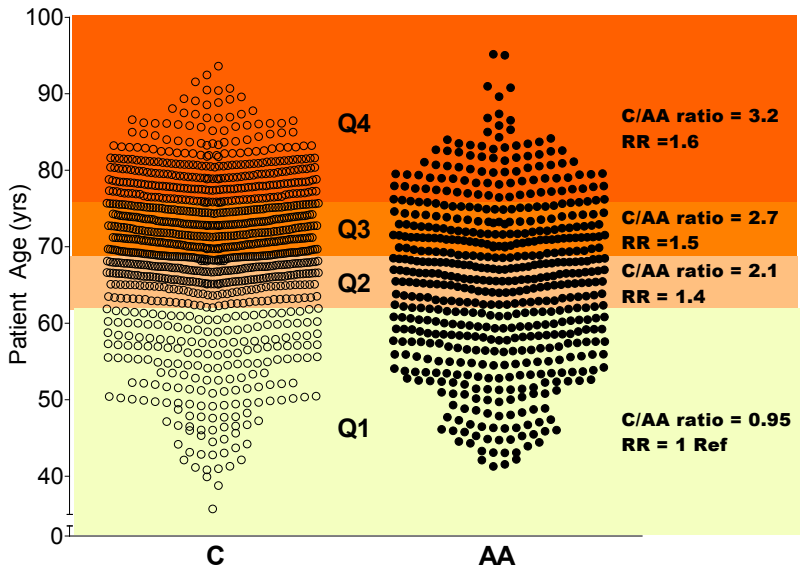

Figure 1: Mushroom Cloud Plots

Recorded ages of all patients analyzed in this study are shown ( 1 circle $=1$ patient). Quartiles of pooled data were generated, and univariable analysis performed using Poisson regression. Each quartile is delineated with color shading to illustrate the increasing number of Caucasian patients $(C)$ versus African-American (AA) within that particular quartile. RR=Relative Risk.

\section{Graphical Presentation of Results}

Shown below are two novel approaches for presenting our findings using next generation graphics (Figures 1 and 2). These examples illustrate the interpretability and effectiveness of displaying relative risk results as a multidimensional plot of the data.

\section{Perspectives and Conclusions}

An important objective of research is being able to effectively present one's data in an informative yet interesting and aesthetically pleasing fashion. Informative visualization goes beyond revealing associations in the data that otherwise would not be revealed upon a cursory glance at data tables. While our data has focused on using these

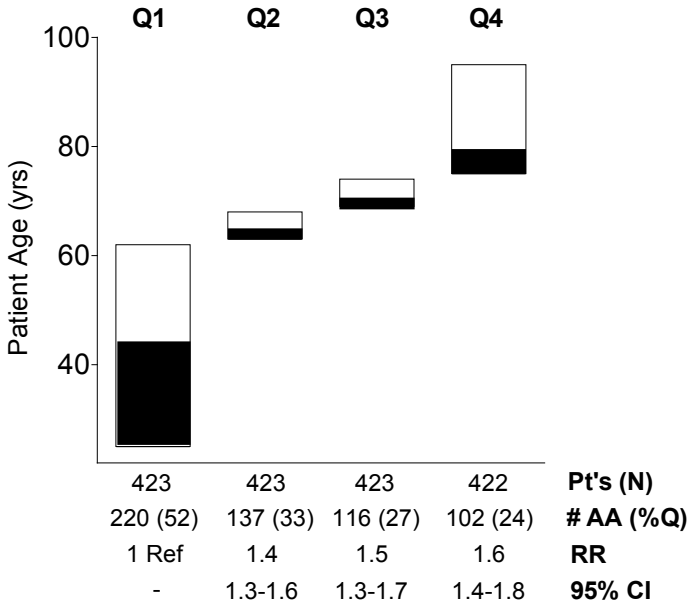

Figure 2: Floating-Bar Plot bar provides visual representation of the numbers that are shown below the plot, illustrating the Caucasian/African-American ratio within that particular quartile. RR=Relative Risk, with $95 \%$ confidence interval $(\mathrm{Cl})$.

techniques in the field of cancer research they are easily generalizable to other fields of study.

\section{Acknowledgements}

Approval was obtained from the Institutional Review Board at the Brody School of Medicine. We would like to thank Dr. Robert G. Sawyer MD and Hope Landrine PhD for their support and encouragement of this work.

\section{Funding}

This manuscript was supported by grant R21HL098780 (E.J.A.) from National Institutes of Health.

\section{References}

1. United states census bureau. State \& county quick facts. 2010;2013 